\title{
ADAMTS genes and the risk of cerebral aneurysm
}

\author{
*Astrid Arning, PhD, ${ }^{1}$ Astrid Jeibmann, MD, ${ }^{2}$ Stephan Köhnemann, PhD, ${ }^{3}$ \\ Benjamin Brokinkel, MD, ${ }^{4}$ Christian Ewelt, MD, ${ }^{4}$ Klaus Berger, PhD, ${ }^{5}$ Jürgen Wellmann, PhD, ${ }^{5}$ \\ Ulrike Nowak-Göttl, MD, ${ }^{6}$ Walter Stummer, MD, ${ }^{4}$ Monika Stoll, PhD, ${ }^{1}$ and Markus Holling, MD ${ }^{4}$

\begin{abstract}
${ }^{1}$ Institute of Human Genetics, Genetic Epidemiology; ${ }^{5}$ nstitute of Epidemiology and Social Medicine, University of Münster; ${ }^{2}$ Institute of Neuropathology; ${ }^{3}$ nnstitute of Legal Medicine; ${ }^{4}$ Department of Neurosurgery, University Hospital Münster; and ${ }^{6}$ Institute of Clinical Chemistry, Thrombosis and Hemostasis Treatment Center, Kiel, University Hospital Schleswig-Holstein, Kiel, Germany
\end{abstract}

OBJECTIVE Cerebral aneurysms (CAs) affect $2 \%-5 \%$ of the population, and familial predisposition plays a significant role in CA pathogenesis. Several lines of evidence suggest that genetic variations in matrix metalloproteinase genes (MMP) are involved in the etiopathology of CAs. The authors performed a case-control study to investigate the effect of 4 MMP variants from the ADAMTS family on the pathogenesis of CAs.

METHODS To identify susceptible genetic variants, the authors investigated 8 single nucleotide polymorphisms (SNPS) in 4 genes from the ADAMTS family (ADAMTS2, -7, -12, and -13) known to be associated with vascular diseases. The study included 353 patients with CAs and 1055 healthy adults.

RESULTS The authors found significant associations between CA susceptibility and genetic variations in 3 members of the ADAMTS family. The largest risk for CA (OR 1.32, $p=0.006)$ was observed in carriers of the ADAMTS2 variant rs11750568, which has been previously associated with pediatric stroke. Three SNPs under investigation are associated with a protective effect in CA pathogenesis (ADAMTS12 variant rs1364044: OR 0.65, $p=0.0001$; and ADAMTS13 variants rs739469 and rs4962153: OR 0.77 and $0.63, p=0.02$ and 0.0006 , respectively), while 2 other ADAMTS13 variants may confer a significant risk ( $r$ 2301612: OR 1.26, $p=0.011$; rs2285489: OR 1.24, $p=0.02$ ).

CONCLUSIONS These results suggest that reduced integrity of the endothelial wall, as conferred by ADAMTS variants, together with inflammatory processes and defective vascular remodeling plays an important role in CA pathogenesis, although the mechanism of action remains unknown. The authors' findings may lead to specific screening of at-risk populations in the future.

http://thejns.org/doi/abs/10.3171/2015.7.JNS154

KEY WORDS cerebral aneurysm; ADAMTS; genes; vascular disorders

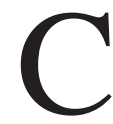
EREBRAL aneurysms (CAs) are dilations of cerebral arteries that affect $2 \%-5 \%$ of the population. ${ }^{35} \mathrm{Al}-$ though most of these lesions are clinically unapparent, CA rupture and subarachnoid hemorrhage usually occur between 40 and 60 years of age. Therefore, early identification of a CA is essential for preventing subsequent bleeding events, which often lead to substantial morbidity and mortality. 4,16
The pathogenesis of CA formation and rupture is complex and seems to arise from multiple factors. A familial predisposition is the strongest risk factor for the development of a CA; siblings of patients with CA are at a 4-fold increased risk of hemorrhage,,$^{40}$ suggesting a significant genetic component to CA pathogenesis. ${ }^{37}$ Environmental factors, such as smoking, hypertension, and extensive alcohol intake, appear to play a more important role in the

ABBREVIATIONS CA = cerebral aneurysm; FDR = false discovery rate; GWAS = genome-wide association study; HWE = Hardy-Weinberg equilibrium; ICB = intracerebral bleeding; IS = ischemic stroke; MI = myocardial infarction; MMP = matrix metalloproteinase; PS = pediatric stroke; SAB = subarachnoidal bleeding; SNP = single nucleotide polymorphism; TSR = thrombospondin Type 1 sequence repeat motif.

SUBMITTED January 2, 2015. ACCEPTED July 14, 2015.

INCLUDE WHEN CITING Published online January 8, 2016; DOI: 10.3171/2015.7.JNS154.

* Drs. Arning and Jeibmann contributed equally to this work. 
rupture of CAs..$^{10,11,17,26,37}$ The development and rupture of aneurysms involve local degradation of vascular structural proteins and remodeling of the diseased blood vessels. Elastin and collagens are major structural components of the vascular wall, and it has been suggested that elastinolysis is particularly pertinent to vessel dilation, while collagenolysis relates to aneurysm rupture. ${ }^{45}$

In recent years, compelling evidence has indicated that a number of matrix metalloproteinases (MMPs), which possess proteolytic activity against extracellular matrix proteins such as elastin and collagens, play an important role in the disease etiopathology of various types of aneurysms. ${ }^{45}$

Although the genetic basis of CA is poorly understood, the identification of susceptible genetic variants may increase our understanding of CA formation and rupture mechanisms. Extensive genetic association studies are urgently needed to elucidate the molecular pathogenesis of CAs. ${ }^{46}$

In recent years, candidate gene association studies have been the most common approach to identify susceptibility genes for complex diseases such as CA. However, our ability to identify relevant candidates is generally restricted by our understanding of disease pathophysiology, which in many cases is limited. For CA, several physiological pathways, including inflammation, 5,15,33,36 disruption of the extracellular matrix surrounding the arterial wall, ${ }^{38}$ and numerous heritable connective tissue disorders, ${ }^{39}$ have been implicated in disease occurrence. However, the conventional candidate gene approach has been widely disappointing for CA, and most findings have not been replicated. ${ }^{21,29}$ Once MMP participation in vascular remodeling became apparent, researchers proposed that variations in $M M P$ may play a role in CA pathogenesis. ${ }^{12,21,24} \mathrm{In}$ addition, subsequent results from a genome-wide association study (GWAS) for pediatric stroke patients have highlighted a crucial role for various ADAMTS (A-disintegrin and metalloproteinase with thrombospondin motifs) metalloproteinases in disease pathophysiology. ${ }^{1}$

The ADAMTS proteins are members of a metalloprotease family that contain the thrombospondin type 1 sequence repeat motifs common to extracellular matrix proteins. ${ }^{27}$

Members of the ADAMTS family play a role in extracellular matrix degradation and are involved in several other biological processes, including coagulation, angiogenesis, and cell migration. ${ }^{13}$ Variations within several ADAMTS genes have been implicated in prothrombotic, ${ }^{13}$ inflammatory, ${ }^{22,28}$ and connective tissue diseases, ${ }^{42}$ suggesting a potential role in CA pathogenesis..$^{18}$ Together, these findings suggest that ADAMTS genes may be excellent screening candidates for CA pathogenesis; however, to our knowledge, it remains unknown if and where these genes act on disease susceptibility.

To address whether variations in ADAMTS genes are genetic determinants of CAs, we analyzed 8 variants of 4 ADAMTS genes associated with diverse vascular phenotypes in 353 patients with CAs and 1055 healthy adults, all from Northwest Germany.

\section{Methods \\ Patients}

Our study included 258 patients harboring a CA who were treated at the Department of Neurosurgery, University Hospital Muenster. From these 258 patients, we identified 222 probands (79.4\% with ruptured aneurysms and $20.6 \%$ with unruptured aneurysms) who were considered eligible for genotyping after assessment of DNA quality. All study participants were investigated with respect to clinical parameters. To enhance the power of our study, we also included in our analysis 131 CA patients who experienced either subarachnoidal bleeding (SAB) or intracerebral bleeding (ICB) from a 2010 independent genetic association study initiated at the Institute of Epidemiology and Social Medicine in Muenster. In all patients (9.07\% of whom had multiple aneurysms), the diagnosis of CA was made using cerebral digital subtraction angiography and confirmed at surgery. Healthy adults served as the control group $(n=1055)$ and were recruited from the population-based Dortmund Health Study, which was conducted in the same region..$^{31,41,43}$ All recruitment and research protocols were reviewed and approved by the institutional review board of the University of Muenster, and written informed consent was obtained from all study participants.

\section{Single Nucleotide Polymorphism Selection, Genotyping, and Quality Control}

DNA was extracted from peripheral blood using standard methods. For genotyping, we selected 8 single nucleotide polymorphisms (SNPs) recently shown to be associated with pediatric stroke (rs469568, rs11750568, rs1364044), ${ }^{1}$ ischemic stroke (rs2285489, rs739469, rs2301612, rs4962153), ${ }^{13}$ and myocardial infarction (rs4380028; Table 1). ${ }^{34}$

For genotyping, we used 2 ng genomic DNA and Taqman SNP Genotyping Assays (Applied Biosystems, Inc.), following the manufacturers' instructions.

Genotyping efficiency was $>98 \%$, and allele identification was performed manually using SDS2.3 software. All laboratory analyses were performed at the Institute of Arteriosclerosis Research in Muenster.

All patients in the control group were already genotyped using the Infinium HD 2.5 M Omni Chip (Illumina, Inc.) and analyzed with GenomeStudio. For SNPs with no directly genotyped data available, we inputted genotypes using the genome-analysis software PLINK V 1.06. ${ }^{32}$

To ensure the comparability of our results, we replicated the genotyping of all variants and showed a significant association with CA using Taqman SNP Genotyping Assays, following the manufacturers' instructions.

\section{Statistical Analysis}

We compared allele frequencies of the risk-associated variants in patients with $\mathrm{CA}$ and in the control group by using the chi-squared test, as analyzed in PLINK V1.06. ${ }^{32}$ We used Haploview $3.0^{2}$ to verify that the observed genotype distributions were in Hardy-Weinberg equilibrium (HWE).

We applied logistic regression models to examine the 
TABLE 1. Overview of genetic variants and their associated diseases

\begin{tabular}{cllll}
\hline Chromosome & Gene & \multicolumn{1}{c}{ SNP } & Associated Disease & Reference \\
\hline 5 & ADAMTS12 & rs1364044 & Pediatric stroke & Arning et al., 2012 \\
\hline 5 & ADAMTS2 & rs11750568 & Pediatric stroke & Arning et al., 2012 \\
\hline 5 & ADAMTS2 & rs469568 & Pediatric stroke & Arning et al., 2012 \\
\hline 9 & ADAMTS13 & rs2285489 & Ischemic stroke & Hanson et al., 2009 \\
\hline 9 & ADAMTS13 & rs739469 & Ischemic stroke & Hanson et al., 2009 \\
\hline 9 & ADAMTS13 & rs2301612 & Ischemic stroke & Hanson et al., 2009 \\
\hline 9 & ADAMTS13 & rs4962153 & Ischemic stroke & Hanson et al., 2009 \\
\hline 15 & ADAMTS7 & rs4380028 & Myocardial infarction & Reilly et al., 2011 \\
\hline
\end{tabular}

association between each SNP and the occurrence of a CA in an additive model of inheritance that included testing trends across SNP categories. All models were adjusted for age and sex. Since 8 SNPs were genotyped, we used the false discovery rate control method to correct for multiple testing, which was analyzed with PLINK V1.06. ${ }^{32}$

\section{Results}

To determine if genetic variations within the ADAMTS family could affect a patient's susceptibility for developing a CA, we analyzed 8 polymorphisms within 4 ADAMTS genes in 353 patients with CA and in 1055 adults in the control group.

Women represented the majority of patients (67\%), and we found no significant differences in mean age (56.7 vs 54.5 years) between the CA group and the control group.

We genotyped 8 candidate SNPs previously associated with ischemic stroke, ${ }^{13}$ myocardial infarction, ${ }^{34}$ or pediatric stroke. ${ }^{1}$ The allele frequencies for each SNP and the odds ratio (OR) for each genotype are reported in Table 2. No deviation from the HWE was observed for any of the 8 SNPs.

Five of the 8 SNPs under investigation were significantly associated with CA after adjusting for age and sex. The ORs ranged from 0.65 to 1.31 and were similar in magnitude to those previously reported for pediatric ${ }^{1}$ and ischemic ${ }^{13}$ stroke. The remaining 2 SNPs failed to demon- strate a statistically significant association with CA. If the results were adjusted for multiple testing, we would lose 1 of the 6 significant findings.

The largest risk for CA (OR 1.32, $\mathrm{p}=0.006)$ was observed in carriers of the ADAMTS2 variant rs11750568, which was previously associated with pediatric stroke. ${ }^{1}$

\section{Discussion}

In this study we evaluated the relationship between an elevated risk of CA development and the presence of 8 ADAMTS metalloproteinase gene variants already known to be associated with vascular diseases.

Once MMP participation in vascular remodeling became apparent, researchers proposed that MMPs might contribute to CA pathogenesis. ${ }^{12,21,24}$ The role of MMPs in normal tissue remodeling processes and connective tissue turnover has been well established, ${ }^{19,23}$ and increased MMP levels have been associated with several pathological states. To our knowledge, it remains unknown how MMPs act on CA susceptibility. ${ }^{20,30}$

Previous studies found that ADAMTS gene variants were significantly associated with several related phenotypes, ${ }^{1,13,34}$ resulting in this gene family becoming an attractive target for genetic screening in patients with a CA.

In the current study, the largest CA risk associated with a single variant $(\mathrm{OR} 1.31, \mathrm{p}=0.006)$, was observed for carriers of the ADAMTS2 variant rs11750568, an allele

TABLE 2. Summary of association results

\begin{tabular}{cccccccccc}
\hline CHR & SNP & NMISS & Minor Allele & MAF & CHISQ & OR & p Value* & Model $1 \dagger$ & FDR_BH \\
\hline 5 & rs1364044 & 1360 & T & 0.2068 & 13.32 & 0.6524 & $0.0001889 \S$ & $0.0003635 \S$ & $0.001511 \S$ \\
\hline 5 & rs11750568 & 1325 & A & 0.3704 & 7.785 & 1.318 & $0.005919 \S$ & 0.07472 & $0.01578 \S$ \\
\hline 5 & rs469568 & 1401 & A & 0.5014 & 0.8393 & 1.086 & 0.3517 & 0.958 & 0.4019 \\
\hline 9 & rs2285489 & 1382 & T & 0.4056 & 4.955 & 1.241 & $0.02158 \S$ & $0.03018 \S$ & $0.02923 \S$ \\
\hline 9 & rs739469 & 1405 & $\mathrm{C}$ & 0.3821 & 3.091 & 0.7672 & $0.02192 \S$ & $0.05004 \S$ & $0.02923 \S$ \\
\hline 9 & rs2301612 & 1384 & $\mathrm{C}$ & 0.506 & 6.458 & 1.257 & $0.01088 \S$ & $0.04544 \S$ & $0.02177 \S$ \\
\hline 9 & $\mathrm{rs} 4962153$ & 1390 & $\mathrm{~A}$ & 0.1209 & 11.62 & 0.6323 & $0.0006033 \S$ & $0.00412 \S$ & $0.002413 \S$ \\
\hline 15 & $\mathrm{rs} 4380028$ & 1366 & $\mathrm{~T}$ & 0.3841 & 0.4571 & 0.9391 & 0.4998 & 0.6065 & 0.4998 \\
\hline
\end{tabular}

$\mathrm{CHISQ}=$ chi-squared; $\mathrm{CHR}=$ chromosome; $\mathrm{MAF}=$ minor allele frequency; NMISS = null or missing SAS values.

* Analyzed by logistic regression.

$\dagger p$ value adjusted for age and sex.

$\ddagger p$ value after adjustment using the false discovery rate as described by Benjamini-Hochberg

$\S$ Statistically significant. 
previously associated with pediatric stroke. ${ }^{1}$ Mutations in the ADAMTS2 gene have been also connected to the Ehlers-Danlos syndrome, a severe connective tissue disorder. ${ }^{43,43}$ Schievink and colleagues proposed over 20 years ago that this kind of tissue disease enhances the potential for developing a CA. ${ }^{39}$ This hypothesis has been supported by more current findings, which indicate that mutations in genes that affect the integrity of the extracellular matrix surrounding the arterial wall play an important role in CA vulnerability. ${ }^{3,38}$ In addition, several biomarkers for connective tissue disorders have been identified that are clearly linked to CA pathogenesis. ${ }^{44}$

Moreover, we have identified significant associations between CA susceptibility and genetic variants for 2 other members of the ADAMTS family. The ADAMTS12 variant rs1364044 seems to offer a protective effect against CA pathogenesis, while the ADAMTS13 variants rs2285489 and rs2301612 confer a significant risk for developing CAs.

Interestingly, ADAMTS12 seems to be involved in the degradation of cartilage oligomeric matrix proteins and has been shown to possess antiangiogenic and tumor-suppressive properties ${ }^{9}$ as well as a modest association with heart failure. ${ }^{25}$ Furthermore, subsequent analysis suggested an important role for ADAMTS12 in inflammatory processes. $^{22}$

Inflammation has been frequently connected to CA pathogenesis. ${ }^{3,8,15,33}$ This is primarily because extensive inflammatory and immunological reactions are common in unruptured CAs and may be related to aneurysm formation and rupture. ${ }^{8}$ These observations have been further supported in findings by Hashimoto and colleagues, who identified abnormal vascular remodeling mediated by inflammatory cells to be a key pathologic component of various vascular diseases. They postulated that CAs evolve from a combination of inflammatory processes, hemodynamics, and vascular remodeling. ${ }^{15}$

ADAMTS13, also known as von Willebrand factor cleaving protease, is already established as a pivotal component of blood coagulation, ${ }^{7}$ prothrombotic diseases, ${ }^{13}$ and vascular inflammation. ${ }^{6}$

Recent findings by Hanson et al. confirm an association between the ADAMTS13 variant rs2285489 and CA susceptibility ${ }^{14}$ in a previous study, the same authors reported an association between this variant and a decreased risk of ischemic stroke. ${ }^{13}$

Taken together, our findings emphasize the theory that genetic variants often confer susceptibility to more than 1 disease and that different diseases share common pathological pathways.

Nevertheless, we are aware that our study has several limitations. First, the limited number of individuals included in our study (353 patients with CA and 1055 healthy controls) limits the statistical power and may increase the risk of Type I (false positive) and Type II statistical errors (missed association). This is particularly relevant given the lack of replication of our findings in an independent cohort. However, all genetic variants analyzed in the current study originated from GWAS for related phenotypes. Second, we acknowledge that reproducing previous findings for pediatric ${ }^{1}$ and ischemic ${ }^{13}$ stroke and myocardial infarction ${ }^{34}$ in our study may be considered "pseudoreplication." A recent study reported an association between ADAMTS13 genetic variants and aneurysmal subarachnoid hemorrhage in 183 patients and 680 control patients, which may be considered an independent association in a comparable clinical phenotype. ${ }^{14}$ However, additional studies with larger sample sizes are warranted to generalize our findings. Ideally, these studies should also include additional clinical subphenotypes, i.e., number of aneurysms, the location thereof, and relevant risk factors such as smoking habits, to allow for more detailed, stratified analysis and to refine the observed associations.

\section{Conclusions}

The mechanisms through which ADAMTS gene variants lead to the development of CAs remain unknown. However, our results give rise to the hypothesis that reduced integrity of the endothelial wall, as conferred by ADAMTS gene defects, together with inflammatory processes and defective vascular remodeling, plays an important role in CA pathogenesis and underscores the findings from previous analyses to CA susceptibility. $3,8,13,15,38$

A better understanding of how these genetic variants modulate CA vulnerability may help to implicate underlying pathways, simplify disease prediction, and prevent resultant bleeding events by specific screening of at-risk populations.

\section{Acknowledgments}

We thank the Innovative Medical Research fund of the University of Münster Medical School for supporting this work.

\section{References}

1. Arning A, Hiersche M, Witten A, Kurlemann G, Kurnik K, Manner D, et al: A genome-wide association study identifies a gene network of ADAMTS genes in the predisposition to pediatric stroke. Blood 120:5231-5236, 2012

2. Barrett JC: Haploview: Visualization and analysis of SNP genotype data. Cold Spring Harb Protoc 2009:pdb.ip71, 2009

3. Bateman JF, Boot-Handford RP, Lamandé SR: Genetic diseases of connective tissues: cellular and extracellular effects of ECM mutations. Nat Rev Genet 10:173-183, 2009

4. Bederson JB, Awad IA, Wiebers DO, Piepgras D, Haley EC Jr, Brott T, et al: Recommendations for the management of patients with unruptured intracranial aneurysms: A Statement for healthcare professionals from the Stroke Council of the American Heart Association. Stroke 31:2742-2750, 2000

5. Chalouhi N, Ali MS, Jabbour PM, Tjoumakaris SI, Gonzalez LF, Rosenwasser RH, et al: Biology of intracranial aneurysms: role of inflammation. J Cereb Blood Flow Metab 32:1659-1676, 2012

6. Chauhan AK, Kisucka J, Brill A, Walsh MT, Scheiflinger F, Wagner DD: ADAMTS13: a new link between thrombosis and inflammation. J Exp Med 205:2065-2074, 2008

7. Chauhan AK, Motto DG, Lamb CB, Bergmeier W, Dockal M, Plaimauer B, et al: Systemic antithrombotic effects of ADAMTS13. J Exp Med 203:767-776, 2006

8. Chyatte D, Bruno G, Desai S, Todor DR: Inflammation and intracranial aneurysms. Neurosurgery 45:1137-1147, 1999

9. El Hour M, Moncada-Pazos A, Blacher S, Masset A, Cal 
S, Berndt S, et al: Higher sensitivity of Adamts12-deficient mice to tumor growth and angiogenesis. Oncogene 29:30253032,2010

10. Foroud T: Whole exome sequencing of intracranial aneurysm. Stroke 44 (6 Suppl 1):S26-S28, 2013

11. Foroud T, Koller DL, Lai D, Sauerbeck L, Anderson C, Ko $\mathrm{N}$, et al: Genome-wide association study of intracranial aneurysms confirms role of Anril and SOX17 in disease risk. Stroke 43:2846-2852, 2012

12. Galis ZS, Khatri JJ: Matrix metalloproteinases in vascular remodeling and atherogenesis: the good, the bad, and the ugly. Circ Res 90:251-262, 2002

13. Hanson E, Jood K, Nilsson S, Blomstrand C, Jern C: Association between genetic variation at the ADAMTS13 locus and ischemic stroke. J Thromb Haemost 7:2147-2148, 2009

14. Hanson E, Olsson S, Bayazit B, Csajbok LZ, Nylén K, Nellgård B, et al: Association between variation in ADAMTS13 and aneurysmal subarachnoid hemorrhage. Thromb Res 131:99-101, 2013

15. Hashimoto T, Meng H, Young WL: Intracranial aneurysms: links among inflammation, hemodynamics and vascular remodeling. Neurol Res 28:372-380, 2006

16. International Study of Unruptured Intracranial Aneurysms Investigators: Unruptured intracranial aneurysms - risk of rupture and risks of surgical intervention. N Engl J Med 339:1725-1733, 1998 (Erratum in N Engl J Med 340:744, 1999)

17. Kleinloog R, van 't Hof FN, Wolters FJ, Rasing I, van der Schaaf IC, Rinkel GJ, et al: The association between genetic risk factors and the size of intracranial aneurysms at time of rupture. Neurosurgery 73:705-708, 2013

18. Le Goff C, Cormier-Daire V: The ADAMTS(L) family and human genetic disorders. Hum Mol Genet 20 (R2):R163R167, 2011

19. Malemud CJ: Matrix metalloproteinases (MMPs) in health and disease: an overview. Front Biosci 11:1696-1701, 2006

20. Maradni A, Khoshnevisan A, Mousavi SH, Emamirazavi SH, Noruzijavidan A: Role of matrix metalloproteinases (MMPs) and MMP inhibitors on intracranial aneurysms: a review article. Med J Islam Repub Iran 27:249-254, 2013

21. McColgan P, Thant KZ, Sharma P: The genetics of sporadic ruptured and unruptured intracranial aneurysms: a genetic meta-analysis of 8 genes and 13 polymorphisms in approximately 20,000 individuals. J Neurosurg 112:714721,2010

22. Moncada-Pazos A, Obaya AJ, Llamazares M, Heljasvaara R, Suárez MF, Colado E, et al: ADAMTS-12 metalloprotease is necessary for normal inflammatory response. J Biol Chem 287:39554-39563, 2012

23. Moore CS, Crocker SJ: An alternate perspective on the roles of TIMPs and MMPs in pathology. Am J Pathol 180:12-16, 2012

24. Morris DR, Biros E, Cronin O, Kuivaniemi H, Golledge $\mathrm{J}$ : The association of genetic variants of matrix metalloproteinases with abdominal aortic aneurysm: a systematic review and meta-analysis. Heart 100:295-302, 2014

25. Morrison AC, Felix JF, Cupples LA, Glazer NL, Loehr LR, Dehghan A, et al: Genomic variation associated with mortality among adults of European and African ancestry with heart failure: the cohorts for heart and aging research in genomic epidemiology consortium. Circ Cardiovasc Genet 3:248-255, 2010

26. Nahed BV, Bydon M, Ozturk AK, Bilguvar K, Bayrakli F, Gunel M: Genetics of intracranial aneurysms. Neurosurgery 60:213-226, 2007

27. Nicholson AC, Malik SB, Logsdon JM Jr, Van Meir EG: Functional evolution of ADAMTS genes: evidence from analyses of phylogeny and gene organization. BMC Evol Biol 5:11, 2005

28. Paulissen G, El Hour M, Rocks N, Guéders MM, Bureau F, Foidart JM, et al: Control of allergen-induced inflammation and hyperresponsiveness by the metalloproteinase ADAMTS-12. J Immunol 189:4135-4143, 2012

29. Peck G, Smeeth L, Whittaker J, Casas JP, Hingorani A, Sharma P: The genetics of primary haemorrhagic stroke, subarachnoid haemorrhage and ruptured intracranial aneurysms in adults. PLoS One 3:e3691, 2008

30. Peters DG, Kassam A, St Jean PL, Yonas H, Ferrell RE: Functional polymorphism in the matrix metalloproteinase-9 promoter as a potential risk factor for intracranial aneurysm. Stroke 30:2612-2616, 1999

31. Pfaffenrath V, Fendrich K, Vennemann M, Meisinger $\mathrm{C}$, Ladwig KH, Evers S, et al: Regional variations in the prevalence of migraine and tension-type headache applying the new IHS criteria: the German DMKG Headache Study. Cephalalgia 29:48-57, 2009

32. Purcell S, Neale B, Todd-Brown K, Thomas L, Ferreira MA, Bender D, et al: PLINK: a tool set for whole-genome association and population-based linkage analyses. Am J Hum Genet 81:559-575, 2007

33. Rahme RJ, Bendok BR: A role for inflammation in intracranial aneurysm pathophysiology? Neurosurgery 66:N14-N15, 2010

34. Reilly MP, Li M, He J, Ferguson JF, Stylianou IM, Mehta NN, et al: Identification of ADAMTS7 as a novel locus for coronary atherosclerosis and association of $\mathrm{ABO}$ with myocardial infarction in the presence of coronary atherosclerosis: two genome-wide association studies. Lancet 377:383-392, 2011

35. Rinkel GJ, Djibuti M, Algra A, van Gijn J: Prevalence and risk of rupture of intracranial aneurysms: a systematic review. Stroke 29:251-256, 1998

36. Roder C, Kasuya H, Harati A, Tatagiba M, Inoue I, Krischek B: Meta-analysis of microarray gene expression studies on intracranial aneurysms. Neuroscience 201:105-113, 2012

37. Ruigrok YM, Rinkel GJ: Genetics of intracranial aneurysms. Stroke 39:1049-1055, 2008

38. Ruigrok YM, Rinkel GJ, van't Slot R, Wolfs M, Tang S, Wijmenga C: Evidence in favor of the contribution of genes involved in the maintenance of the extracellular matrix of the arterial wall to the development of intracranial aneurysms. Hum Mol Genet 15:3361-3368, 2006

39. Schievink WI, Björnsson J, Piepgras DG: Coexistence of fibromuscular dysplasia and cystic medial necrosis in a patient with Marfan's syndrome and bilateral carotid artery dissections. Stroke 25:2492-2496, 1994

40. Schievink WI, Wijdicks EF, Piepgras DG, Chu CP, O'Fallon WM, Whisnant JP: The poor prognosis of ruptured intracranial aneurysms of the posterior circulation. $\mathbf{J}$ Neurosurg 82:791-795, 1995

41. Vennemann MM, Hummel T, Berger K: The association between smoking and smell and taste impairment in the general population. J Neurol 255:1121-1126, 2008

42. Wang WM, Lee S, Steiglitz BM, Scott IC, Lebares CC, Allen ML, et al: Transforming growth factor-beta induces secretion of activated ADAMTS-2. A procollagen III N-proteinase. J Biol Chem 278:19549-19557, 2003

43. Winter AC, Hoffmann W, Meisinger C, Evers S, Vennemann M, Pfaffenrath V, et al: Association between lifestyle factors and headache. J Headache Pain 12:147-155, 2011

44. Yurt A, Vardar E, Selçuki M, Ertürk AR, Ozbek G, Atçi $\mathrm{B}$ : Biomarkers of connective tissue disease in patients with intracranial aneurysms. J Clin Neurosci 17:1119-1121, 2010

45. Zhang B, Dhillon S, Geary I, Howell WM, Iannotti F, Day IN, et al: Polymorphisms in matrix metalloproteinase-1, -3 , 
-9 , and -12 genes in relation to subarachnoid hemorrhage. Stroke 32:2198-2202, 2001

46. Zhang J, Claterbuck RE: Molecular genetics of human intracranial aneurysms. Int J Stroke 3:272-287, 2008

\section{Disclosures}

The authors report no conflict of interest concerning the materials or methods used in this study or the findings specified in this paper.

\section{Author Contributions}

Conception and design: Holling, Arning, Jeibmann, Köhnemann, Berger, Wellmann, Nowak-Göttl, Stummer, Stoll. Acquisition of data: Holling, Arning, Köhnemann, Brokinkel, Ewelt. Analysis and interpretation of data: Holling, Arning, Jeibmann, Brokinkel, Ewelt, Wellmann, Nowak-Göttl, Stoll. Drafting the article: Holling, Arning, Jeibmann, Berger, Stoll. Critically revising the article: Holling, Arning, Jeibmann, Köhnemann, Ewelt, Berger, Wellmann, Stummer, Stoll. Reviewed submitted version of manuscript: Holling, Brokinkel, Ewelt, Stummer, Stoll. Approved the final version of the manuscript on behalf of all authors: Holling. Statistical analysis: Holling, Arning, Jeibmann, Wellmann, Nowak-Göttl, Stoll. Administrative/technical/material support: Holling, Köhnemann, Berger, Nowak-Göttl, Stummer, Stoll. Study supervision: Holling, Arning, Stummer.

\section{Correspondence}

Markus Holling, Department of Neurosurgery, University Hospital Münster, Albert-Schweitzer-Campus 1A, Münster 48149, Germany. email: hollingm@ukmuenster.de. 